\title{
Prefrontally Driven Downregulation of Neural Synchrony Mediates Goal-Directed Forgetting
}

\author{
Simon Hanslmayr, ${ }^{1,2,3}$ Gregor Volberg, ${ }^{1}$ Maria Wimber, ${ }^{4}$ Nora Oehler, ${ }^{2}$ Tobias Staudigl, ${ }^{2}$ Thomas Hartmann, ${ }^{2}$ \\ Markus Raabe, ${ }^{1}$ Mark W. Greenlee, ${ }^{1}$ and Karl-Heinz T. Bäuml ${ }^{1}$ \\ ${ }^{1}$ Department of Experimental Psychology, University of Regensburg, 93053 Regensburg, Germany, ${ }^{2}$ Department of Psychology and ${ }^{3 Z u k u n f t s k o l l e g,}$ \\ University of Konstanz, 78457 Konstanz, Germany, and ${ }^{4}$ Medical Research Council Cognition and Brain Sciences Unit, Cambridge CB2 7EF, United \\ Kingdom
}

Neural synchronization between distant cell assemblies is crucial for the formation of new memories. To date, however, it remains unclear whether higher-order brain regions can adaptively regulate neural synchrony to control memory processing in humans. We explored this question in two experiments using a voluntary forgetting task. In the first experiment, we simultaneously recorded electroencephalography along with fMRI. The results show that a reduction in neural synchrony goes hand-in-hand with a BOLD signal increase in the left dorsolateral prefrontal cortex (dIPFC) when participants are cued to forget previously studied information. In the second experiment, we directly stimulated the left dIPFC with repetitive transcranial magnetic stimulation during the same task, and show that such stimulation specifically boosts the behavioral forgetting effect and induces a reduction in neural synchrony. These results suggest that prefrontally driven downregulation of long-range neural synchronization mediates goal-directed forgetting of long-term memories.

\section{Introduction}

Memories are thought to be stored within synaptic connections among widespread cortical networks (Fuster, 1997), with the strength of these connections being modified by neural synchrony (Markram et al., 1997). Phase synchronization establishes communication between distant brain areas (Fries, 2005), presumably shaping neural plasticity by facilitating long-term potentiation (Buzsáki, 2006). Consistently, previous electrophysiological studies in humans have reported enhanced phase synchronization in memory tasks to be associated with memory formation (Fell et al., 2001; Summerfield and Mangels, 2005; Fell and Axmacher, 2011). However, it is unknown whether long-range synchronization can be regulated by higher-order brain regions in a voluntary, task-relevant manner. We here investigate the impact of the prefrontal cortex on neural synchrony during voluntary forgetting.

Although forgetting is usually viewed as a failure of memory, it can help us to remove outdated or unwanted information to free up memory capacity, rendering our memory system flexible and adaptive (Bjork, 1989; Levy and Anderson, 2002). Indeed, people can intentionally forget episodic memories when cued to do so, as is shown in the directed-forgetting task (Bjork, 1970). In the list

Received April 12, 2012; revised Aug. 20, 2012; accepted Aug. 25, 2012.

Author contributions: S.H. and K.-H.T.B. designed research; S.H., G.V., N.O., and T.S. performed research; M.W., T.H., M.R., and M.W.G. contributed unpublished reagents/analytic tools; S.H. and G.V. analyzed data; S.H., M.W., and K.-H.T.B. wrote the paper.

Parts of this research have been supported by a grant from the Deutsche Forschungsgemeinschaft (Project HA 5622/1-1) awarded to S.H. We thank Anna Karmann, Stefanie Birkner, and Marina Koepfer for help with data acquisition.

Correspondence should be addressed to Dr. Simon Hanslmayr, Department of Psychology, University of Konstanz, Box ZPR, 78457 Konstanz, Germany. E-mail: simon.hanslmayr@uni-konstanz.de.

DOI:10.1523/JNEUROSCI.1777-12.2012

Copyright $\odot 2012$ the authors $\quad 0270-6474 / 12 / 3214742-10 \$ 15.00 / 0$ method of this task, participants study two lists of items and receive a cue to either forget or continue to remember the preceding list (Fig. 1a). On a later memory test, participants are asked to recall all of the previously presented items, including to-be-forgotten items. During study of the first list, participants do not know whether the items of the list are to be forgotten later. Even so, the forget cue impairs recall of the items of this list, reflecting goal-directed forgetting of the obsolete List 1 information. A previous study found directed forgetting to be reflected by a sustained decrease in phase synchronization in the upper alpha-lower beta frequency range $(11-13 \mathrm{~Hz})$ during second-list encoding (Bäuml et al., 2008). Additionally, the forget cue also improves recall of the new, second-list items, a behavioral effect that has been linked to stimulus-induced alpha power decreases (Bäuml et al., 2008; Pastötter et al., 2008). Consistent with the cognitive literature, this improvement effect therefore appears to be dissociable from the forgetting effect (Sahakyan and Delaney, 2003; Pastötter and Bäuml, 2010).

Lesion and fMRI studies suggest that memory control depends on the engagement of the dorsolateral PFC (dlPFC) (Conway and Fthenaki, 2003; Anderson et al., 2004; Depue, 2012). Because voluntary forgetting requires control over contents in memory, we performed two experiments to test whether the dorsolateral PFC mediates voluntary forgetting specifically by downregulating long-range neural synchrony. In the first experiment, EEG was recorded simultaneously with fMRI while participants performed a directed-forgetting task. In the second experiment, repetitive transcranial magnetic stimulation (rTMS), a technique used to directly stimulate specific brain regions, was applied at the dlPFC during the same forgetting task while recording the EEG. If indeed dlPFC downregulates neural synchrony to induce 
a Remember/Forget Run

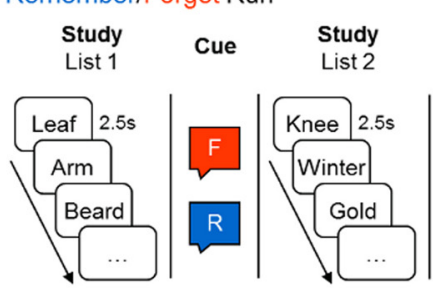

b

6 Remember Runs +6 Forget Runs

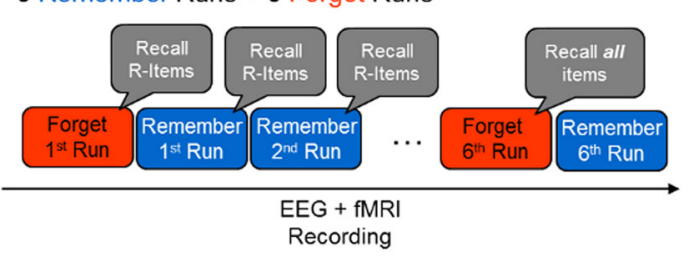

C Behavioral Results (6th Run)

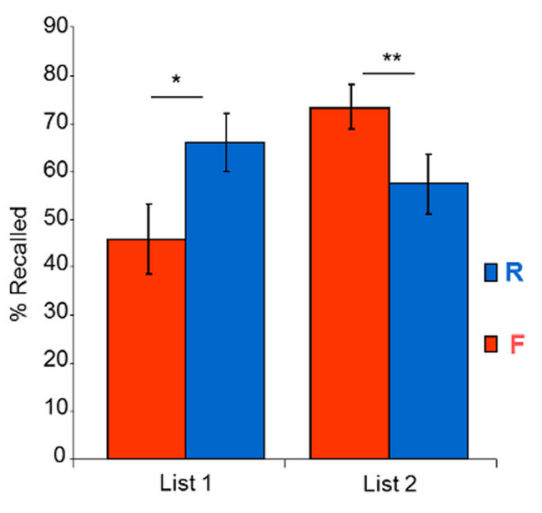

Figure 1. $\quad \boldsymbol{a}$, The directed-forgetting task is shown. $\boldsymbol{b}$, The procedure as used in the current study is presented. Participants performed repeated runs of the directed-forgetting task, with a pseudorandom order of conditions, such that participants did not know whether the first-list items would be required for later recall. Only the last forget (sixth) run was followed by a surprise memory test, in which participants were asked to recall all items of the current run, including to-be-forgotten items. c, Behavioral results of the last run are plotted. Error bars indicate SES. ${ }^{*} p<0.05,{ }^{* *} p<$ 0.01 .

voluntary forgetting, (1) enhanced activity in the dlPFC should go hand-in-hand with a reduction in neural synchrony, and (2) stimulation of the dIPFC should specifically modulate neural synchrony and the behavioral forgetting effect.

\section{Materials and Methods}

Participants (Experiments 1 and 2). In the first (EEG-fMRI) experiment, 22 healthy participants (mean age, 23.05 years; range, 20-29; 7 males) remained in the analysis after excluding 2 participants due to a too high number of EEG artifacts. In the second (rTMS-EEG) experiment, 44 participants (mean age, 22.2; range, 18-28; 18 males) remained in the analysis, after excluding 4 participants due to poor EEG data. Subjects were randomly assigned to the dlPFC or vertex (control) stimulation group, with 22 subjects remaining in each group (dlPFC: mean age, 21.95 years; range, 18-28; 10 males; vertex: mean age, 22.41 years; range, $18-$ 27; 8 males). All participants gave their written informed consent, and the experimental protocol was approved by the local ethical review board.

Material and procedure (Experiments 1 and 2). As study material, 240 words were drawn from the Medical Research Council Psycholinguisitic Database (Coltheart, 1981) and translated into German. The word material was split into 24 lists of 10 words each. The lists were matched according to word frequency (mean, 52.95; SD, 51.12), number of letters (mean, 5.36; SD, 1.15), syllables (mean, 1.69; SD, 0.54), concreteness (mean, 542.9; SD, 42.5), and imageability (mean, 563.24; SD, 32.3). Each of the 24 lists was equally often used across the four conditions (ForgetList 1, Forget-List 2, Remember-List 1, and Remember-List 2).

A schematic depiction of the directed-forgetting task is shown in Figure $1 a$. Within each run (Remember or Forget), two lists of 10 words each were presented sequentially on a computer screen, which was visible to the participants via a mirror attached to the head coil. The words were written in black on a gray background. Each word was presented for $2.5 \mathrm{~s}$, preceded by a fixation cross with a variable duration of 1.5-2.5 s. After List 1 , a cue was presented to either continue remembering the words, or to forget the words from this list. The cue was shown for $5 \mathrm{~s}$ and was followed by the presentation of the second list of words (same timing parameters as List 1). The second list was always followed by a cue to remember the list. Thereafter, a visual feature detection task was performed as a distracter task $(\sim 3 \mathrm{~min})$, during which arrays of randomly oriented Gabor patches were presented. One-half of the arrays contained a path of 10 collinearly oriented Gabor elements, and the task was to indicate whether or not an array contained a Gabor path (Field et al., 1993). Finally, a free recall test was performed in which participants were asked to recall all to-be-remembered items of the current run, consistent with the forget instruction. Only in the last run of the Forget condition, participants were asked to recall the words from both lists, including those that they had been instructed to forget (see Fig. $1 b$ ). Whether the Forget or the Remember condition was performed in the last run of the experiment was counterbalanced across participants. Each free recall test was followed by a $30 \mathrm{~s}$ resting period, which served as an fMRI baseline for task-related activity. In the first experiment, verbal responses were digitally recorded using a MRI-compatible microphone (MR confon), and the scanner noise was later removed from the resulting audio files using the free software Audacity (http://audacity.sourceforge.net/). In the second experiment, the verbal responses were manually recorded by the experimenter outside of the EEG booth (transmitted via the intercom).

$f M R I$ recording and analysis (Experiment 1). Imaging was performed using a $3 \mathrm{~T}$ MR head scanner (Siemens Allegra). For the functional series, 2226-2286 whole-brain volumes, consisting of 34 axial slices, were continuously acquired using an interleaved, standard $\mathrm{T} 2{ }^{*}$-weighted echoplanar imaging sequence [time repetition (TR), $2000 \mathrm{~ms}$; time echo (TE), $30 \mathrm{~ms}$; flip angle, $90^{\circ}$; $64 \times 64$ matrices; in-plane resolution, $3 \times 3 \mathrm{~mm}$; slice thickness, $3 \mathrm{~mm}]$. High-resolution sagittal T1-weighted images were acquired after the functional scans, using a MP-RAGE (TR, $2250 \mathrm{~ms}$; TE, $2.6 \mathrm{~ms} ; 1 \mathrm{~mm}$ isotropic voxel size) to obtain a 3D structural scan.

Image preprocessing and statistical analysis was performed using SPM5 (Wellcome Department of Cognitive Neurology, London, UK; www.fil.ion.ucl.ac.uk/spm), running under MATLAB (MathWorks). After discarding the first few images of each session, time series were corrected for differences in slice acquisition time, unwarped, and spatially realigned to the first image of the session. The mean functional image was coregistered with the structural image, which was then normalized to a Montreal Neurological Institute (MNI) (www.mni.mcgill.ca) template in standard stereotactic space. The resulting normalization parameters were applied to all functional images, which were subsequently smoothed with a Gaussian kernel of $8 \mathrm{~mm}$ (FWHM).

fMRI data were recorded and analyzed in a blocked manner. The single-subject hemodynamic responses were modeled by convolving a boxcar function covering the duration of each word list or resting period with a first-order canonical hemodynamic response function (Friston et al., 1995). The resulting time series were then used as regressors in a voxelwise, fixed-effects general linear model. The resulting data were high-pass filtered at $256 \mathrm{~s}$. Time series were modeled by four blocked covariates, corresponding to the encoding periods of the experimental conditions Forget-List 1, Forget-List 2, Remember-List 1, and Remember-List 2, and a further blocked covariate corresponding to the resting periods. Covariates modeling the free recall periods, the distracter task, 
Table 1. Results of the exploratory whole-brain analysis

\begin{tabular}{lrllllll}
\hline & \multicolumn{7}{l}{ MNI coordinates } \\
\cline { 2 - 7 } Anatomical label & BA & HS & Size & $x$ & $y$ & $z$ & $t$ \\
\hline Forget $>$ Remember & & & & & & & \\
MFG & & & & & & & \\
$\quad$ Middle frontal gyrus & 9 & $\mathrm{~L}$ & 137 & -45 & 6 & 39 & 4.32 \\
$\quad$ Middle frontal gyrus & 6 & $\mathrm{~L}$ & & -24 & 0 & 48 & 3.90 \\
$\quad$ Superior frontal gyrus & 6 & $\mathrm{~L}$ & & -30 & 21 & 57 & 3.55 \\
IFG & & & & & & & \\
Inferior frontal gyrus & 46 & $\mathrm{~L}$ & 128 & -45 & 27 & 21 & 4.56 \\
$\quad$ Inferior frontal gyrus & 45 & $\mathrm{~L}$ & & -51 & 30 & 12 & 4.15 \\
$\quad$ Middle frontal gyrus & 9 & $\mathrm{~L}$ & & -35 & 21 & 30 & 3.59 \\
\hline
\end{tabular}

Peak locations of significant differences between the Forget and Remember condition during List 2 encoding ( $F>$ $\left.R ; p_{\text {corr }}<0.05\right)$. No significant differences were observed in the $F<R$ contrast.

session-specific effects, and movement parameters determined during realignment were also included in the model.

On a single-subject level, the four conditions of interest (Forget-List 1, Forget-List 2, Remember-List 1, Remember-List 2) were contrasted separately against the resting periods. $T$ maps derived from these comparisons were then entered into a second-level full-factorial ANOVA, with the factors CONDITION (Forget vs Remember) and LIST (List 1 vs List 2). Planned comparisons within this model were conducted between the Forget and Remember condition during List 2 encoding, using one-sided $t$ tests ( $p<0.001$, uncorrected). Analysis was focused on an anatomically defined region of interest (ROI) of the dorsolateral PFC (BA 9 and BA 46), using the WFU PickAtlas toolbox (www.fmri.wfubmc.edu/cms/ software). Only clusters within this region that survived a cluster-level correction $(p<0.05)$ are reported. Cluster-level correction refers to whether finding a significant cluster with a given extent of $k$ (or more) voxels, at a given threshold for the whole search volume $(p<0.001)$, would be expected by chance (in the present dataset, $k=20$ voxels). A cluster-level-corrected $p$ value of 0.05 denotes that the likelihood of finding an equally sized (or bigger sized) cluster by chance is $5 \%$. Results of an exploratory whole-brain analysis with the same statistical threshold $(p<$ 0.001 , uncorrected; cluster-level correction, $p<0.05)$ are reported in Table 1.

Psychophysiological interaction analysis (Experiment 1). To investigate connectivity on the fMRI data level, psychophysiological interaction (PPI) analysis was performed (Friston et al., 1997). This analysis permits investigation of the correlation between two physiological variables (e.g., BOLD signal in two brain regions) and its interaction with a psychological variable (Forget vs Remember). Following our results from the simple contrasts (Forget $>$ Remember), BA 9 was taken as a seed region. Four subjects had to be excluded from this analysis because they either did not show significant activation of this region ( $\mathrm{F}>\mathrm{R} ; p<0.05$, uncorrected; $N=2$ ), or the clusters of activation were too remote from the cluster obtained on the group level $(>4 \mathrm{~cm} ; N=2)$. Before the PPI analysis, the fMRI data was concatenated across the three sessions to allow simultaneous extraction of the dlPFC time series across all sessions. For each subject, an $8 \mathrm{~mm}$ sphere was built around a cluster showing the maximal activation $(F>R$ ) in the dlPFC (BA 9). If more than one significant cluster was obtained, the cluster closest to the peak voxel obtained in the group analysis was taken (MNI coordinates: $-45,6,39$ ). The mean distance from this peak voxel across subjects was $1.15 \mathrm{~cm}(\mathrm{SD}$, 0.64). PPI analysis was performed as implemented in SPM5. The main purpose of this analysis was to investigate the interaction of dlPFC activity with hippocampal activity during voluntary forgetting, as suggested by a prior fMRI study (Anderson et al., 2004). We therefore restricted the statistical analysis to an anatomically defined ROI comprising the left and right hippocampus (using WFU Pickatlas).

EEG recording and preprocessing (Experiment 1). The EEG was recorded inside the scanner using an MR-compatible 64-channel EEG system (Brain Products). Sixty-two channels were used to record scalp EEG and were mounted in an elastic cap (EasyCap) positioned according to the international 10-10 system. FCz was used as reference electrode, and impedances were kept below $20 \mathrm{k} \Omega$. Note that the MR-compatible electrode caps have an inbuilt impedance of $5 \mathrm{k} \Omega$. Vertical eye movements were recorded with an additional channel placed below the left eye, and the electrocardiogram (ECG) was recorded by an electrode placed below the left scapula to facilitate off-line removal of cardioballistic artifacts. The signals were amplified between 0.1 and $100 \mathrm{~Hz}$, with a notch filter at $50 \mathrm{~Hz}$. The EEG data were sampled at $5000 \mathrm{~Hz}$, and the clock of the EEG amplifier was synchronized to the clock output of the MR scanner using a "SynchBox" device, manufactured by Brain Products, to facilitate offline removal of the MR gradient artifact.

When the EEG is recorded inside the MR scanner, the data are contaminated by (1) the MR gradient artifact and (2) the cardioballistic artifact, which have to be removed by various preprocessing steps. Both artifacts were removed using the FMRIB plug-in for EEGLAB (Delorme and Makeig, 2004; Niazy et al., 2005), running under MATLAB (MathWorks).

The first artifact is generated by the switching of the MR gradient each time a new image is collected. To remove this artifact, a template was constructed separately for each MR gradient artifact and for each EEG channel. The exact onset of the artifact was known by triggers delivered from the MR scanner every time a new volume was acquired. The average template was then subtracted from the actual artifact. For template construction, a moving average of 21 neighboring images and a linear combination of the major principal components describing the residual artifacts were used. These were determined automatically by means of sorted eigenvalues. The corrected data were then downsampled to $500 \mathrm{~Hz}$ and high-pass-filtered (using a FIR filter) at $0.5 \mathrm{~Hz}$. Bad stretches of data in the continuous EEG, due to incomplete gradient artifact removal or other artifacts, were identified and removed by careful visual inspection.

The second artifact, the cardioballistic artifact, is generated by heartbeats that show a characteristic deflection in the ECG electrode denoted as QRS complex (Debener et al., 2008). For detection of the QRS onsets, the algorithm implemented in the FMRIB plug-in was used. This algorithm performs a temporal principal component analysis separately for each EEG channel. The first three components were taken as an optimal basis set for describing the artifact shape, amplitude, and scale. This set was fitted to and then subtracted from each artifact occurrence. As with the removal of the MR gradient artifacts, this was performed separately for each channel.

In a last step, the cleaned EEG data were subjected to an infomax independent component analysis (ICA) to correct for residual artifacts. Main sources of artifacts were eyeblinks, eye movements, tonic muscle activity, as well as residual cardioballistic and gradient artifacts. Components that corresponded to one of these artifacts were identified by visual inspection and removed. The remaining components were then backprojected into EEG signal space. The data were then segmented into epochs ranging from -2500 to $2500 \mathrm{~ms}$ relative to word onset. Before EEG analysis, the single trials were visually inspected and rejected if they contained residual artifacts. An average of 41.4 (range, 28-54), 44.3 (range, 28-57), 39.4 (range, 19-55), and 42.6 (range, 24-56) trials remained for analysis of the four conditions Forget-List 1, Forget-List 2, Remember-List 1, and Remember-List 2, respectively.

EEG recording and preprocessing (Experiment 2). EEGs were recorded from 128 electrodes with active shielding mounted in an elastic cap with an equidistant montage (ANT; www.ant-neuro.com). Signals were recorded in a shielded booth with a DC amplifier (ANT), with a sampling rate of $2048 \mathrm{~Hz}$. Preprocessing was performed using Fieldtrip (http:// www.ru.nl/neuroimaging/fieldtrip) (Oostenveld et al., 2011). The data during List 2 encoding were epoched into segments of $975 \mathrm{~ms}$, timelocked to the TMS pulse (25-1000 ms after TMS). The first $25 \mathrm{~ms}$ were discarded to eliminate the TMS artifact. Thereafter, the data were corrected for blinks and eye movements, using ICA. Remaining artifacts, due to muscle activity or poor EOG correction, were excluded by careful visual inspection. Before phase-locking analysis, the data were downsampled to $512 \mathrm{~Hz}$.

rTMS procedure (Experiment 2). An overview of the experimental procedure is shown in Figure 6a. TMS pulses were delivered with a Magstim Rapid2 stimulator via a figure-of-eight-shaped air film cooled coil (magstim; www.magstim.com). To ensure that the TMS pulses were stimulating the target brain region with high anatomical precision, TMS was 
guided by a neuronavigation system, which coregisters the individual MRI with the position of the TMS coil using a 3D tracking device (ANTVisor; www.ant-neuro.com). Individual high-resolution T1-weighted MRIs were acquired from a Philips 1.5 T scanner (T1-TFE; TR, 10.656 $\mathrm{ms}$; TE, $4.99 \mathrm{~ms}$; flip angle, $8^{\circ}$; $1 \mathrm{~mm}$ isotropic voxel size). The coordinates for dIPFC stimulation were derived from the peak voxel showing the strongest effect in BOLD signal in our previous EEG-fMRI experiment (MNI coordinates: $x=-45, y=6, z=39$; see Fig. 3). The MNI coordinates for vertex stimulation were as follows: $x=0, y=-10, z=$ 80, following Miranda et al. (2006). TMS pulses were delivered at a rate of $1 \mathrm{~Hz}$ during encoding of List 2 items after both forget and remember instructions. There was no systematic temporal coupling between the delivery of the TMS pulses and the List 2 items. A total of 45 TMS pulses at an intensity of $90 \%$ of the individual resting motor threshold were applied during the full length of List 2 encoding, thus leading to a duration of $45 \mathrm{~s}$ of $1 \mathrm{~Hz}$ rTMS stimulation.

Analysis of phase synchronization (Experiments 1 and 2). Phase synchronization here refers to synchrony between distant brain regions as reflected in synchrony between electrode sites. For both experiments, the same analysis was performed using in-house MATLAB scripts. For timefrequency analysis, the EEG epochs were subjected to a Gabor transformation, transforming a signal into a complex time-frequency signal, from which the phase information can be extracted. The data were filtered in a frequency range of $4-45 \mathrm{~Hz}$. The filter parameter for timefrequency resolution (gamma) was set to 1 for the lower frequency range ( $4-20 \mathrm{~Hz}$ ), and to $\pi$ for the higher frequency range $(20-45 \mathrm{~Hz})$, ensuring that the time-frequency characteristics of the different frequency bands are optimally detected.

Before phase synchrony calculation, a current source density transformation was applied to the EEG data using the CSD toolbox (http:// psychophysiology.cpmc.columbia.edu/Software/CSDtoolbox/index.html). This was done to minimize effects of volume conduction (Lachaux et al., 1999). The phase synchrony values were calculated following the procedure of Lachaux et al. (1999) using the phase estimates from the Gabor transformation. This procedure delivers a value that ranges from 0 to 1 , indicating maximal phase variability and maximal phase synchrony, respectively. Phase synchronization values were calculated for all possible pairs of electrodes in a frequency range from 4 to $45 \mathrm{~Hz}$. Because trial numbers can bias phase synchronization measures, we checked whether there was a significant difference between trial numbers across the four conditions, by means of a Friedman ANOVA. This analysis revealed no significant difference in trial numbers across the four conditions. Additionally, a control analysis was conducted in which the number of trials were equated, by means of randomly selecting the minimum amount of available trials. This control analysis revealed similar significant effects $\left(p_{\text {corr }}<0.05\right)$. One specific hypothesis was formulated for the upper alpha-lower beta frequency range as derived from a prior EEG study (Bäuml et al., 2008), but statistical comparisons were calculated across the whole frequency range to determine the specificity of this effect.

For the second (rTMS-EEG) experiment, the analysis was focused on phase coupling in a frequency range between 11 and $18 \mathrm{~Hz}$ informed by the results of Experiment 1. Furthermore, the analysis was restricted to the time window of 500-1000 ms after the TMS pulse, because TMS at the prefrontal cortex induces a strong evoked response in the beta range (lasting $\sim 400 \mathrm{~ms}$ ), which is likely to overshadow more subtle cognitive effects (Rosanova et al., 2009). For this analysis, the number of trials was equated across the two conditions by randomly selecting a minimum number of trials that was available across the two conditions. On average, 194 (range, 55-242) and 199 (83-256) trials remained for the dlPFC and Vertex groups, respectively. Note that the trials in this experiment had shorter epochs $(500 \mathrm{~ms})$ and were time-locked to the TMS pulse leading to higher trial numbers than in the first experiment.

Analysis of EEG power (Experiment 1). Before analysis of oscillatory power modulation $\left(\mu \mathrm{v}^{2}\right)$, the EEG data were re-referenced to average reference. For time-frequency analysis, a Gabor transformation with the same parameters as for the phase synchrony analysis was used. To quantify event-related power changes, the percentage of poststimulus power change in relation to a prestimulus baseline period was calculated. The baseline period was set to $-0.5 \mathrm{~s}$ to stimulus onset for the lower fre- quency range $(4-20 \mathrm{~Hz})$, and to $-0.25 \mathrm{~s}$ to stimulus onset for the higher frequency range $(20-45 \mathrm{~Hz})$. Before statistical analysis, the signal change values were collapsed to obtain six frequency bands: theta $(4-8 \mathrm{~Hz})$, alpha $(9-11 \mathrm{~Hz})$, beta $1(12-19 \mathrm{~Hz})$, beta $2(20-29 \mathrm{~Hz})$, and gamma $(30-45 \mathrm{~Hz})$; and four time windows: $0-0.5,0.5-1.0,1.0-1.5$, and $1.5-$ $2.0 \mathrm{~s}$. Based on prior studies, specific hypotheses were formulated with respect to the alpha frequency band. Statistical comparisons, however, were conducted across all frequency bands to investigate the specificity of the effects.

EEG-fMRI analysis (Experiment 1). Combined EEG-fMRI analysis was performed to investigate whether the experimentally induced effects on the BOLD signal and brain oscillations covaried on a block-by-block level. The toolbox MARSBAR (http://marsbar.sourceforge.net) was used to first extract the fMRI signal from the preprocessed EPI images, averaged across all voxels constituting the left dlPFC region of interest. To isolate the signal corresponding to a given condition (Forget-List 2 and Remember-List 2, respectively), these time series were then fit with the condition regressors, that is, the predicted time courses in the GLM design matrix. A mean value for each block was obtained by averaging across those segments of the fitted time series that corresponded to the duration of a block of interest, as defined by the predicted time course turning positive at the beginning of a block, and negative at the end of a block. This procedure resulted in six values for the Remember condition, and six values for the Forget condition. In a last step, these values were $z$-transformed to account for global signal differences between participants.

To obtain the mean levels of phase synchronization in each block, the same procedure as in the study by Hanslmayr et al. (2007) was applied. This procedure estimates the mean level of phase synchrony for each block by calculating the single-trial phase deviation from the mean phase, applying the circular variance procedure proposed by Fisher (1993). These single-trial phase deviation values were then collapsed across those electrode pairs, time points ( -2.0 to $2.0 \mathrm{~s}$ ), and frequency bands (11-18 $\mathrm{Hz}$ ), in which significant effects emerged. To obtain the level of phase synchrony for each block, the phase deviation values were collapsed across artifact-free single trials within a block. For each block, a maximum of 10 single trials was available; however, $<10$ trials were available in most of the cases due to artifacts. If less than five single trials were available, the block was discarded. Otherwise, the median across artifactfree single trials was taken as an estimate of the average phase synchronization within a block. Two participants were excluded from the combined EEG-fMRI analysis because less than three blocks remained for analysis of one of the two conditions of interest (Forget-List 2, Remember-List 2). For the remaining 20 participants, an average of 5.5 and 5.3 blocks remained for the Forget and Remember conditions, respectively. Before correlation with the BOLD signal, the data were $z$-transformed to normalize the data across subjects. $Z$-transformation was performed such that the mean level of BOLD/phase synchrony was subtracted from the individual values of the respective blocks, which were then divided by the SD of these values.

Statistical analysis (Experiments 1 and 2). For statistical comparisons of all behavioral data, $t$ tests with the $p$ level set to 0.05 were used. These tests were performed one-sided when a directed hypothesis was tested (e.g., testing for the forgetting and beneficial effects), and two-sided when no directed hypothesis was tested (e.g., testing for the effects of TMS on behavior).

Analysis of the EEG data in both experiments was fully based on nonparametrical Wilcoxon signed-rank tests (one-sided), and a randomization approach was used to correct for multiple comparisons (Hanslmayr et al., 2009a,b). This procedure was originally proposed by Blair and Karniski (1993) and works as follows: First, Wilcoxon's tests were calculated for each electrode pair to investigate how many pairs show a significant difference between the two conditions at a given $p$ level $(p<0.005$, one-sided). Second, 2000 randomization runs were performed in which the conditions were swapped randomly across participants. This procedure produces a distribution of the number of electrodes exceeding a certain statistical threshold ( $p<0.005$; in our case) under the null hypothesis (no systematic difference between conditions), and evaluates whether a given number of electrode pairs exhibiting a significant differ- 
ence between two conditions at this threshold can be expected by chance. If the $p$ value $\left(p_{\text {corr }}\right)$ of this randomization test is $<0.05,<5 \%$ of the permutation runs exhibited equal or more electrode pairs with a significant difference between the two conditions.

fMRI comparisons were conducted using one-sided $t$ tests $(p<0.001$, uncorrected), with the anatomically defined ROI of the dIPFC (BA 9 and BA 46). Only clusters within this ROI surviving cluster-level correction $(p<0.05)$ are reported as significant (see Table 1 for a whole-brain analysis). For statistical analysis of the correlations between the BOLD signal in the left dlPFC and phase synchronization, we followed a random-effects approach. One correlation coefficient (Spearman) was calculated to fit the data points (maximum, 6) of each single subject. These correlation coefficients were then Fisher $z$ transformed. Thereafter, the mean (Fisher $z$-transformed) correlation coefficient across all subjects was tested against zero using one-sample $t$ tests (one-sided). To examine the difference in BOLD-phase synchronization correlations between conditions, a dependent-samples $t$ test was performed.

\section{Results}

\section{Experiment 1: simultaneous EEG-fMRI}

Behavioral results

Participants performed several runs of the directed-forgetting task (Fig. 1a,b). Each cue condition was repeated six times, in an unpredictable order. In the first five runs of the Forget condition, subjects were asked to recall only to-be-remembered (List 2) items at test, which is consistent with the instruction (Fig. 1b). Only in the last (sixth) run of the Forget condition, a surprise test was conducted, in which subjects were asked to recall all words of the current run, including the to-be-forgotten items of List 1 (Fig. $1 b)$. The behavioral data of this sixth run revealed lower recall levels for List 1 items in the Forget condition (45.9\%) compared with the Remember condition $\left(65.9 \%\right.$; $t_{(21)}=-2.15, p<0.05$; Fig. 1c); and they revealed higher recall levels for List 2 items in the Forget condition $(73.2 \%)$ than in the Remember condition $\left(57.3 \% ; t_{(21)}=3.43 ; p<0.005\right.$; Fig. $\left.1 c\right)$. These results demonstrate that subjects were able to voluntarily forget the old, obsolete information, for the benefit of improved memory for the new, relevant information.

To rule out that the forgetting effect was caused by output order effects at test (i.e., the prior recall of the to-be-remembered items), average output positions were calculated for each subject [for details, see Bjork (1970)] and correlated with forgetting. This correlation was close to zero $(r=0.09 ; p>0.5)$, suggesting that the forgetting in this experiment was not caused by differences in output order, which is consistent with prior work showing that output order has a negligible effect on directed forgetting (Geiselman et al., 1983). We additionally analyzed the intrusion rates for the to-be-forgotten List 1 items for the first five runs. Consistent with the literature (Pastötter and Bäuml, 2007), the mean level of intrusions was very low, $4.45 \%$ (SD, 5.9), suggesting that participants were well able to follow the instruction to only recall tobe-remembered items. The intrusion rates did not change across runs $\left(F_{(4,84)}=0.87 ; p>0.45\right)$, and subjects with low and high levels of intrusions (median split) did not differ with respect to their forgetting and enhancement scores (values of $t_{(20)}<1.67$; values of $p>0.1$ ).

\section{Phase synchrony}

Both prior behavioral and prior EEG work indicate that the mechanisms responsible for the forgetting of the obsolete (List 1) information operate during encoding of the new (List 2) information (Bäuml et al., 2008; Pastötter and Bäuml, 2010) (i.e., after the forget or remember cue had been presented). Consistently, phase synchronization measures between the two cue conditions were contrasted by means of the phase-locking value (PLV)
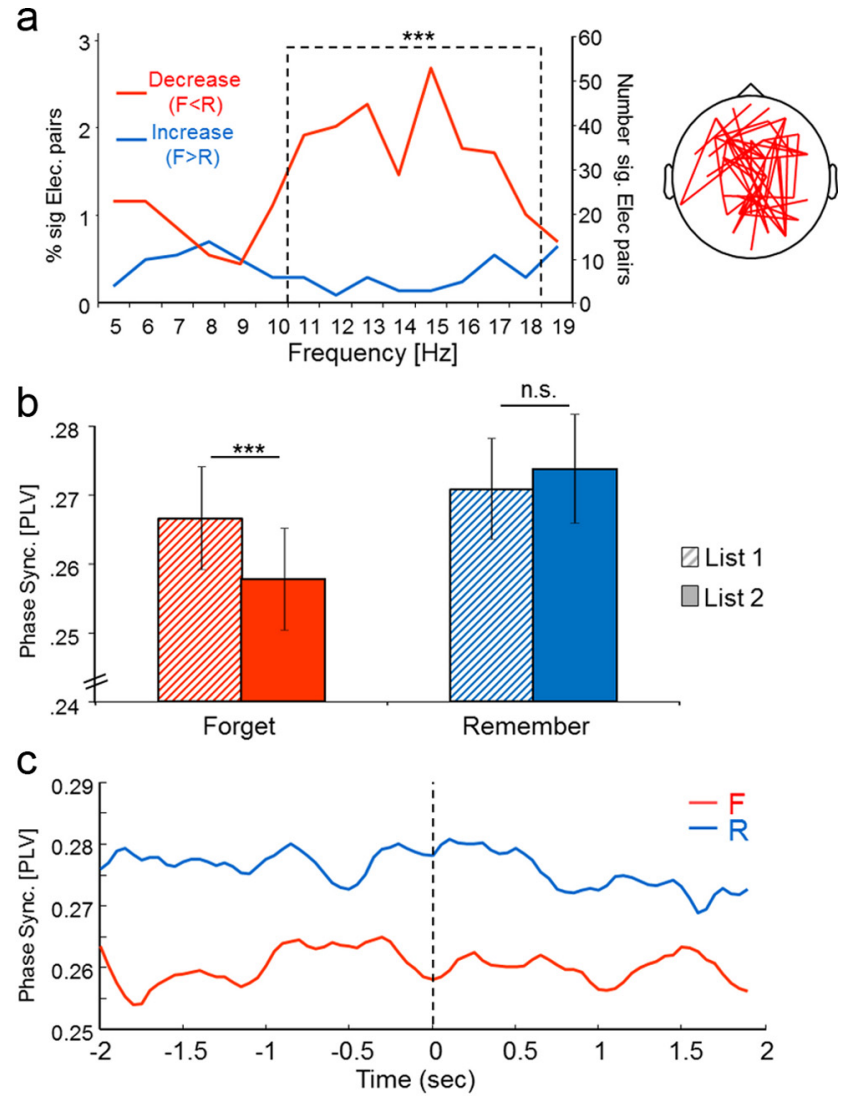

Figure 2. $\quad \boldsymbol{a}$, Differences in phase synchronization between the Forget $(\mathrm{F})$ and the Remember $(\mathrm{R})$ condition are shown across different frequencies ( $x$-axis). The number of electrode pairs showing significant differences $(p<0.005)$ between the two conditions $(F>R / F<R)$ is shown on the $y$-axis. The left axis shows the percentage of significant electrode (sig Elec.) pairings (of all possible pairings), and the right axis shows the absolute number of significant electrode parings. A significant decrease in phase synchronization from 11 to $18 \mathrm{~Hz}$ in the $\mathrm{F}$ compared with the $R$ condition ( $p_{\text {corr }}<0.005$ ) over widespread cortical regions was observed. $\boldsymbol{b}$, Mean levels of phase synchrony are shown for the $F$ and the $R$ conditions during List 1 and List 2 encodings. The pattern shows a decrease across the two lists in the $\mathrm{F}(p<0.005)$ but not in the $\mathrm{R}(p>0.1)$ condition. Error bars indicate SEs. ${ }^{* *} p<0.005$. $c$, The time course of phase synchronization (11-18 Hz) for the electrodes showing a significant difference between the Forget and Remember conditions during List 2 encoding is depicted. Note the tonically reduced phase synchronization in the Forget condition that appears to be independent from stimulus onset.

(Lachaux et al., 1999). In line with our previous study (Bäuml et al., 2008), data were pooled in a peristimulus interval ( -2 to $2 \mathrm{~s}$ ). The results show that the forget cue induced lower levels of phase synchrony than the remember cue $\left(p_{\text {corr }}<0.005\right.$; Fig. $\left.2 a\right)$. This effect was evident over widespread cortical regions in a frequency range within the upper alpha, lower beta band $(11-18 \mathrm{~Hz})$. No differences between the two conditions were found during List 1 encoding, and no changes in phase synchrony in the opposite direction $(\mathrm{F}>\mathrm{R})$ were observed. Further exploration of the data revealed that the forget cue induced a decrease in phase synchronization from List 1 to List $2(Z=-3.72 ; p<0.001)$, whereas no difference between the two lists emerged in the Remember condition $(Z=1.64 ; p>0.1$; Fig. $2 b)$. The difference between List 1 and List 2 phase coupling was significantly higher in the Forget than in the Remember condition $(Z=-3.33$; $p<0.001)$. Examination of the time course of phase synchronization revealed no change between the prestimulus and poststimulus interval, showing that phase synchrony was tonically reduced throughout List 2 encoding, independent of the onset of List 2 items (Fig. 2c). 

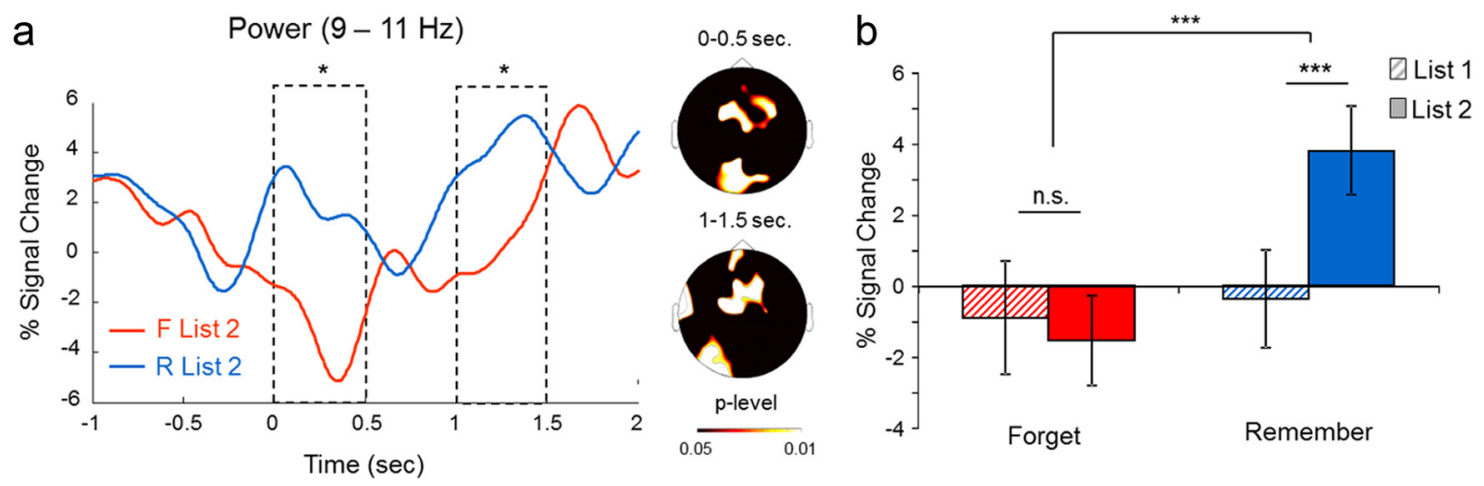

Figure 3. $a$, The time course of alpha power is shown during List 2 encoding for the two conditions. Decreased levels of stimulus induced alpha power in the $F$ compared with the $R$ condition ( $p$ corr $<0.05$ ) were observed $0-500 \mathrm{~ms}$ and $1000-1500 \mathrm{~ms}$ after stimulus presentation over frontal and parietal electrode sites. $\boldsymbol{b}$, Mean levels of alpha power, collapsed across the significant electrode sites and the two time windows, are plotted during List 1 and List 2 encoding. The pattern shows that alpha power increases from List 1 to List 2 in the $R$ condition $(p<0.001)$, whereas no change is observed in the F condition $(p>0.5)$. Error bars indicate SEs. ${ }^{*} p<0.05,{ }^{* * *} p<0.001$.
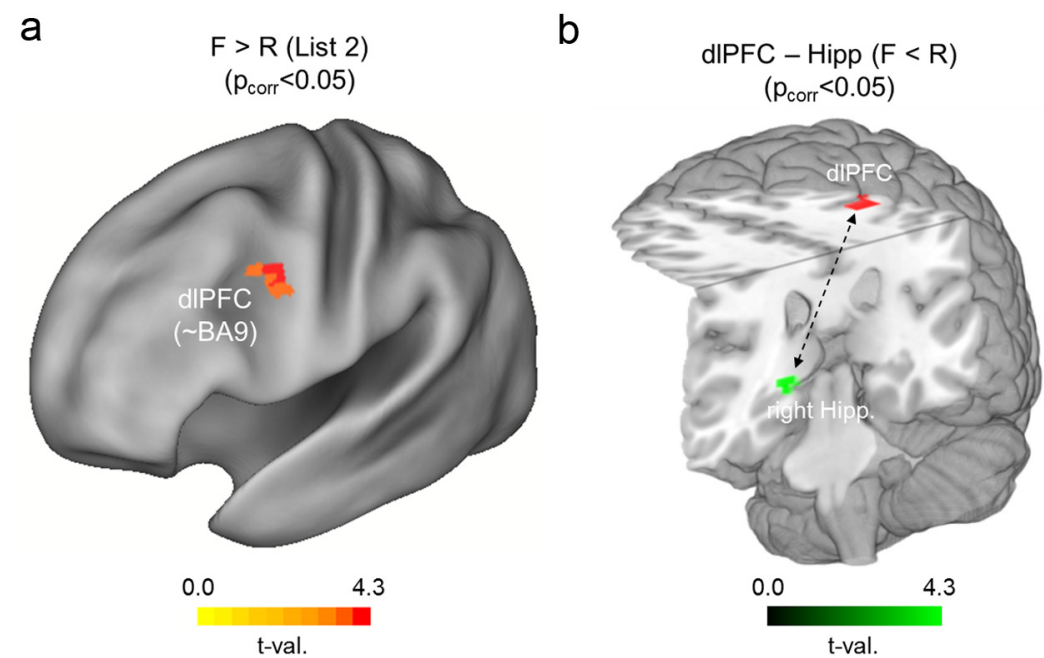

Figure 4. fMRI results are shown. $\boldsymbol{a}$, Voxels demonstrating increased activation in the $F$ compared with the $R$ condition during List 2 study are projected onto an inflated cortical surface $\left(p<0.001\right.$, uncorrected). Significant differences $\left(\mathrm{F}>\mathrm{R} ; p_{\text {corr }}<0.05\right)$ emerged in the left dIPFC (BA 9: $-45,6,39)$. $\boldsymbol{b}$, Results from the connectivity (PPI) analysis are shown. In the Forget condition, connectivity between dIPFC and the right hippocampus was significantly reduced compared with the Remember condition $\left(p_{\text {corr }}<0.05\right)$.

No significant effects emerged in the higher beta $(20-29 \mathrm{~Hz})$ and gamma frequency band $(30-45 \mathrm{~Hz})$. Further analysis indicated that the effects of the forget cue on phase synchronization did not vary across the significant electrode pairs.

\section{EEG power}

Contrasting alpha power between the two conditions during encoding of List 2 items revealed lower levels of stimulus-induced alpha power in the Forget condition, compared with the Remember condition $\left(p_{\text {corr }}<0.05\right.$; Fig. $\left.3 a\right)$. This effect showed a frontoparietal topography and emerged at an early $(0-0.5 \mathrm{~s})$ and a later time window (1-1.5 s). Closer inspection of the effect revealed that alpha power increased from List 1 to List 2 in the Remember condition $(Z=3.12 ; p<0.001)$, but stayed constant in the Forget condition ( $p>0.5$; Fig. $3 b$ ); the difference between the two lists in the Remember condition was significantly larger than in the Forget condition $(Z=2.94 ; p<0.005)$. No significant effects between the two conditions emerged during List 1 encoding, and no effects were obtained in other frequency bands.

\section{fMRI results}

Analogous to the EEG analysis, we contrasted the BOLD signal between the two conditions during study of List 2. Following our hypothesis, the analysis was restricted to an anatomically defined ROI corresponding to the dorsolateral PFC (BA9 and BA46). Higher activity was found in the Forget compared with the Remember condition in the left dlPFC (Fig. 4a; BA 9; MNI coordinates: $-45,6$, 39; voxel size, $\left.21 ; p_{\text {corr }}<0.05\right)$. The results of an exploratory whole-brain analysis are reported in Table 1. No brain region showed higher activity in the Remember compared with the Forget condition, and no differences between conditions were observed during List 1 study.

To investigate the influence of the forget instruction on functional connectivity on the fMRI data level, a PPI analysis was performed. This analysis was conducted in an attempt to replicate a prior fMRI study showing that voluntary memory suppression is also reflected in the interaction between dlPFC and the hippocampus (Anderson et al., 2004). The results of this PPI analysis revealed that the correlation between dlPFC and hippocampus activity was significantly modulated by the forget instruction (Fig. $4 b ; p_{\text {corr }}<0.05$; MNI coordinates: $21,-30,-3$; voxel size, 5 ). This effect was due to a reduced dlPFC-hippocampus connectivity in the Forget (mean $r=-0.002$ ) compared with the Remember condition (mean $r=0.39)$.

\section{Correlation between BOLD and phase synchrony}

The results above suggest that the cue to forget irrelevant memories induced a decrease in phase synchrony along with an activation increase in the left dlPFC. Central to the aim of this study, we investigated whether the decrease in phase synchrony can be predicted by dlPFC activity on a block-by-block basis. To this end, the dlPFC BOLD signal was correlated with phase synchronization during List 2 encoding (for details, see Materials and Methods). To examine whether such a correlation is specifically driven by the experimental condition of interest, correlations across blocks (six blocks per condition) were performed separately for the Forget and Remember conditions. The results revealed that increased activation of the dlPFC correlated with the decrease in phase synchrony in the Forget condition (mean 
$r=-0.33 ; t_{(19)}=-2.19 ; p<0.05$; Fig. $5 a)$, whereas no correlation between dlPFC activation and phase synchronization arose in the Remember condition (Fig. $5 b$; mean $r=0.034 ; t_{(19)}=0.28 ; p>$ $0.50)$; the correlation between dlPFC activity and phase synchrony was significantly higher in the Forget compared with the Remember condition $\left(t_{(19)}=-2.48\right.$; $p<0.05)$. Note that these correlations, although modest in magnitude, are well within the range of EEG-BOLD correlations obtained in other human EEG-fMRI studies (Mantini et al., 2007). Motivated by the results from the fMRI connectivity, we also correlated phase synchrony with the BOLD signal in right hippocampus (Fig. 4b). However, this analysis yielded no significant correlations in any of the two conditions (both values of $p>0.25$ ). It should be noted, however, that the EEG-BOLD correlations were conducted on a block-by-block level with an average of five data points per subject, which is a slight limitation of this analysis. We therefore conducted a second rTMS-EEG experiment to further verify and extend these findings.

\section{Experiment 2:}

\section{simultaneous rTMS-EEG}

The results of the first experiment show that increased activation in the dlPFC goes hand-in-hand with a decrease in neural synchrony during voluntary forgetting. To examine whether this relationship is of a causal more than just a correlational nature, we conducted a combined rTMS-EEG experiment and tested whether direct stimulation of dlPFC has a modulating effect on both the behavioral consequences of the forget instruction and phase synchronization. In this second experiment, 44 participants performed the same forgetting task as in the first experiment. Volunteers were randomly assigned to one of two groups, with one group $(N=22)$ receiving rTMS at dlPFC (MNI coordinates: $-45,6,39)$, and the other group $(N=22)$ receiving rTMS at vertex (a typical control region for TMS studies). A total of 45 TMS pulses with an intensity of $90 \%$ resting motor threshold was delivered at a continuous rate of $1 \mathrm{~Hz}$ during List 2 encoding in both the Remember and Forget conditions (Fig. $6 a$ ).

\section{Behavioral results}

To investigate the impact of rTMS on behavior, the two stimulation site (dlPFC vs vertex) groups were contrasted with respect to their relative forgetting and enhancement scores. The forgetting score was calculated as the difference between List 1 words recalled in the Remember and Forget condition ( $\mathrm{R}$ minus $\mathrm{F}$ ), and the enhancement score was calculated as the difference between List 2 recall in the two conditions (F minus $\mathrm{R}$ ). Analogous to Experiment 1, the behavioral data of the sixth run were analyzed for both enhancement and forgetting scores. The results are shown in Figure 6b. Participants who received rTMS at the

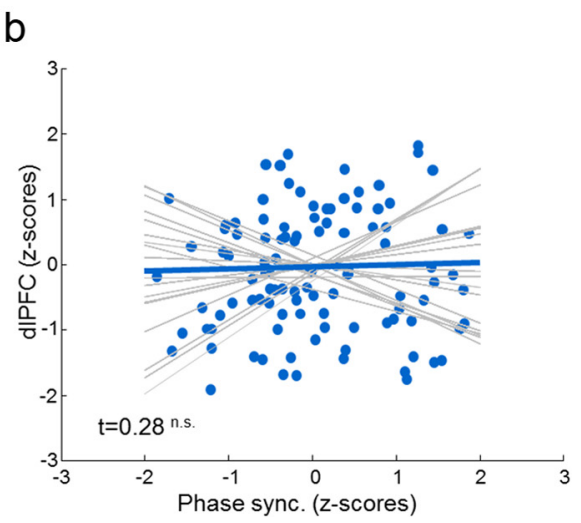

Figure 5. Scatterplots of the single-trial correlations between dIPFC BOLD signal and phase synchronization are shown. $Z$-transformed values of BOLD signal are plotted on the $y$-axis, and $z$-transformed values of phase synchronization are plotted on the $x$-axis. Each dot represents the data of one subject and run during List 2 encoding. The gray lines indicate the fit of the $\boldsymbol{a}, \boldsymbol{b}$, The correlation between dIPFC and phase synchrony is shown for the $\mathrm{F}$ condition $(\boldsymbol{a})$ and for the $\mathrm{R}$ condition $(\boldsymbol{b})$. A robust

a

Figure 6. $\quad \boldsymbol{a}$, The experimental procedure for the combined rTMS-EEG experiment is shown. During study of the second list, subjects received slow rTMS ( 45 pulses) either at dIPFC or at vertex (control site). $\boldsymbol{b}$, The behavioral results of the last run are plotted. Stimulating dIPFC resulted in higher forgetting scores than stimulation at vertex (control). No differences were obtained for enhancement scores. Error bars indicate SEs. ${ }^{*} p<0.05$.

dorsolateral PFC showed a significantly stronger forgetting effect than the control group who received rTMS at vertex (30.0 vs $6.4 \% ; t_{(42)}=2.34 ; p<0.05$, two-tailed). No significant difference was observed for the enhancement effect $\left(6.8 \mathrm{vs} 10.0 \% ; t_{(42)}=\right.$ $-0.45 ; p>0.5)$. Additionally, a two-way ANOVA with the factors GROUP (dlPFC vs vertex) and SCORE TYPE (forgetting vs enhancement) yielded a significant interaction $\left(F_{(1,42)}=4.47\right.$; $p<0.05)$. These results demonstrate that rTMS at the dorsolateral PFC during List 2 encoding selectively boosts voluntary forgetting of List 1 items, without affecting the enhancement of List 2 items. The full pattern of the behavioral results is shown in Table 2, which shows that the increased forgetting scores in the dlPFC group were driven by a decrease in the Forget-List 1 condition, as well as by a moderate increase in the Remember-List 1 condition compared with the vertex group. However, none of these separate comparisons reached significance $\left(t_{(46)}>1.49\right.$; $p>0.1)$.

\section{EEG results}

The effect of rTMS on phase synchronization was examined by contrasting phase synchrony during List 2 encoding between the Forget and the Remember conditions. Following the results of the prior 
Table 2. The average recall scores of the rTMS-EEG experiment are reported separately for each condition

\begin{tabular}{llllll}
\hline \multirow{2}{*}{$\begin{array}{llllll}\text { Stimulation } \\
\text { group }\end{array}$} & List 1 & & & List 2 & \\
\cline { 2 - 3 } \cline { 6 - 7 } Forget & & Remember & & Forget & Remember \\
dIPFC & {$[\%(S D)]$} & {$[\%(S D)]$} & & {$[\%(S D)]$} & {$[\%(S D)]$} \\
Vertex & $45.0(35.9)$ & $73.6(22.6)$ & & $80.0(22.8)$ & $73.3(22.4)$ \\
\hline
\end{tabular}

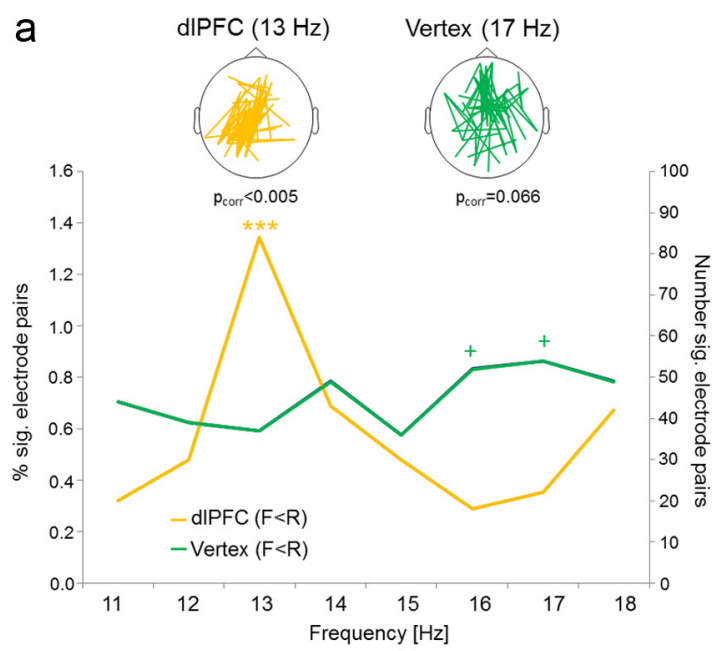

\section{b}

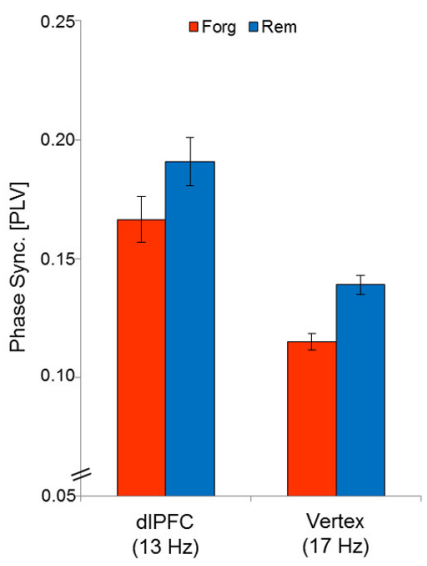

Figure 7. The effects of rTMS on phase synchronization are shown. $\boldsymbol{a}$, Percentage (left) and absolute number of significant (sig.) electrode pairings (right) is shown on the $y$-axis. Participants who received rTMS at dIPFC showed a pronounced decrease in phase synchronization in the Forget compared with the Remember condition at $13 \mathrm{~Hz}$. Participants from the control group, who received rTMS at vertex, showed a marginally significant, but weaker, effect at $17 \mathrm{~Hz}$ in the same direction. Note that the EEG was recorded from 128 channels in this experiment. $\boldsymbol{b}$, The raw PLV scores are shown for the $\mathrm{dPFC}$ and vertex group for those frequency bands and electrode sites that exhibited $\mathrm{F}<\mathrm{R}$ effects. Error bars indicate SEs. ${ }^{* * *} p<0.005,{ }^{+} p<0.1$.

experiment, the analysis was focused on a frequency range of 11-18 $\mathrm{Hz}$. Note that active rTMS was applied in both conditions, thus controlling for unspecific cortical rTMS effects. Participants who were stimulated at the left dlPFC showed a robust decrease in phase synchronization at $13 \mathrm{~Hz}$ in the Forget compared with the Remember condition ( $p_{\text {corr }}<0.005$ ), whereas participants from the control (vertex) group showed only a marginally significant effect at $17 \mathrm{~Hz}$ $\left(p_{\text {corr }}=0.066 ;\right.$ Fig. 7$)$ in the same direction. Importantly, the number of electrode pairs exhibiting a significant reduction in phase synchronization differed significantly between the two groups ( 84 vs 54; $\left.\mathrm{X}^{2}{ }_{1}=6.52 ; p<0.05\right)$, showing stronger phase desynchronization in response to the forget cue in the dIPFC than the control group. No significant effects in the opposite direction $(F>R)$ were observed in any of the two groups.

\section{Discussion}

We investigated whether prefrontal brain regions can regulate neural synchronization during long-term memory control, using a voluntary forgetting task. Using simultaneous EEG-fMRI recordings in the first experiment, we were able to demonstrate that an instruction to forget obsolete memories induces a reduction in long-range phase synchronization along with a BOLD signal increase in the left dorsolateral PFC. Importantly, the BOLD signal increase in the dlPFC correlated with a decrease in neural synchrony, specifically when subjects were asked to forget the previously encoded information. In the second experiment, we showed that direct stimulation of the dIPFC via rTMS selectively boosts voluntary forgetting and phase desynchronization, thus providing evidence for a causal role of dlPFC in driving forgetting and phase synchrony.

These results strongly suggest that prefrontally regulated neural synchrony reflects an active memory control process. Following the idea that memories are represented in widely distributed cortical networks (Fuster, 1997), a widespread decrease in phase synchronization might reflect the downregulation of the cortical network representing the obsolete, to-beforgotten memories. This interpretation is corroborated by our finding that stimulating the dlPFC during List 2 encoding selectively impaired memory for the tobe-forgotten List 1 items, without affecting memory for the to-be-remembered List 2 items. This result is quite remarkable, given that rTMS was applied during List 2 encoding, but fits with prior work indicating that the forget cue induces two dissociable effects (Sahakyan and Delaney, 2003; Bäuml et al., 2008; Pastötter and Bäuml, 2010), which both operate during List 2 encoding (i.e., after the forget cue has been given) (Bäuml et al., 2008; Pastötter and Bäuml, 2010).

These results also replicate prior fMRI and lesion studies indicating that memory control is mediated by the dorsolateral PFC (Conway and Fthenaki, 2003; Anderson et al., 2004; Depue et al., 2007). Although the focus of dlPFC activity in our study is slightly more dorsal compared with the results by Anderson et al. (2004), our findings replicate previous results of Nyberg et al. (2009), reporting activation of a similar region in BA9 during a longterm memory updating task. Like Anderson et al. (2004), we also observed that voluntary forgetting affected BOLD connectivity between dIPFC and hippocampus, even though forgetting was associated with lowered dlPFC-hippocampus connectivity in the present study. Note that, in contrast to the think/no-think paradigm (Anderson and Green, 2001; Anderson et al., 2004), which requires subjects to suppress the retrieval of a previously learned association, the directedforgetting task used in the present study is a long-term memory updating task in which old information has to be forgotten during the learning of new information, likely involving different prefrontal-hippocampal interactions.

In addition to decreased phase coupling, the EEG results of Experiment 1 also revealed increased power in the alpha band specifically when subjects were asked to remember the previously learned information. In contrast, no such changes occurred in the Forget condition (Fig. 3b). Several previous studies linked stimulus-induced alpha power decreases to item-specific information processing and long-term memory encoding (for review, see Hanslmayr et al., 2012). In particular, alpha power has been shown to increase with the amount of encoded information (Sederberg et al., 2006; Pastötter et al., 2008), mirroring decreased encoding quality (Underwood, 1978). In line with these findings, the alpha power decreases might indicate that the forget cue resets the neural activity back to first-list level, thus ensuring high encoding quality for the new information (Pastötter et al., 2008, 2011). This reset view is corroborated by a previous study (Bäuml et al., 2008) showing that the alpha power difference between the 
Forget and Remember condition specifically correlates with improved memory for List 2 items.

Importantly, the effects of the forget cue on alpha power and phase synchrony differed markedly in timing. Whereas the forgetting-induced decrease in phase synchrony was independent of the onset of the List 2 items (Fig. 2c), the decreases in alpha power were time-locked to the stimulus onset. This further suggests that the alpha power decreases reflect item-specific memory encoding processes of the List 2 items, whereas the tonically reduced phase synchronization reflects inhibition of List 1 items.

Regarding the cognitive processes underlying directed forgetting, one dominant view is that the forget cue triggers the inhibition of the original encoding context of the items, creating problems in retrieving these items when tested later (Geiselman et al., 1983; Anderson, 2005; Bäuml et al., 2008). This idea acknowledges the fact that directed forgetting is usually found in recall tasks but not in recognition tasks (Bjork, 1989), and that forgetting can be eliminated once the original encoding context gets reactivated (Bjork and Bjork, 1996; Bäuml and Samenieh, 2010, 2012). An analogy might be that, after directed forgetting, the files are still present on the hard disk, but the paths are temporarily lost. Our results are in line with this assumption, and suggest that reduced phase synchronization might be a correlate of this unbinding process, which impairs access to these items when tested later (Bäuml et al., 2010). Although being speculative, this interpretation fits with the idea that long-range synchrony acts to dynamically bind (and unbind) cortically distributed representations (Varela et al., 2001; Fries, 2005).

Another, noninhibitory, explanation of directed forgetting is the context change account (Sahakyan and Kelley, 2002). This hypothesis assumes that the forget cue induces a change in subjects' mental context between study of the two lists, which then impairs List 1 recall due to a mismatch between the context at encoding and the context at retrieval. Although this noninhibitory view can account for a number of behavioral findings (Sahakyan and Kelley, 2002), it is hard to reconcile with the present findings. In particular, the context change account cannot explain why phase synchronization is reduced below baseline level. Additionally, in a previous EEG study, we investigated the brain oscillatory correlates of the mental context change paradigm, as proposed by Sahakyan and colleagues, and did not find any decreases in phase synchronization during List 2 encoding (Pastötter et al., 2008).

It is also worth highlighting that, despite the fact that the EEG data from the first experiment were recorded inside the MR scanner, typically causing substantial distortions of the raw EEG signal, our finding of decreased phase synchrony in the upper alpha-lower beta frequency band perfectly replicates the results of a previous conventional EEG study (Bäuml et al., 2008). In this former directed-forgetting study, the decrease in phase synchronization specifically predicted the amount of List 1 forgetting, establishing a tight link between decreased levels of phase coupling during List 2 study and the forgetting of obsolete memories. As in this prior study, the effect in phase synchronization showed a widespread topography involving frontal, temporal, and parietal electrode sites. These independent results suggest that reliable EEG data were recorded despite the noisy scanner environment.

Our finding that slow rTMS $(1 \mathrm{~Hz})$ has a facilitatory effect on voluntary forgetting and phase desynchronization is perfectly in line with recent literature showing that slow rTMS applied at the prefrontal cortex can boost neural processing and cognitive performance (Li et al., 2004; Knoch et al., 2006; Ward et al., 2010).
For instance, slow rTMS applied at the dorsolateral prefrontal cortex enhances blood flow and BOLD signal at the stimulated region (Li et al., 2004; Knoch et al., 2006) and has been found to increase cognitive performance in a selective attention task (Ward et al., 2010). This literature seems to be at odds with papers arguing that slow rTMS $(<5 \mathrm{~Hz})$ disturbs neural activity by creating virtual lesions (Chen et al., 1997; Thut and Pascual-Leone, 2010). The exact reasons for why slow rTMS is sometimes facilitatory and sometimes inhibitory are not yet known, but it has been suggested that the effects of rTMS vary depending on the stimulated region (Rosanova et al., 2009), the state of the stimulated region (Silvanto et al., 2008), and on whether rTMS is applied on-line (during task performance) or off-line (before task performance). Likely, future studies are needed to shed more light onto these open issues.

The impact of oscillatory activity on memory formation has long been demonstrated on the microlevel (i.e., in single-cell and multicell recordings in animals) (Buzsáki, 2010). It is now increasingly being recognized that brain oscillations, and largescale phase synchrony in particular, also play a fundamental role for human long-term memory (Fell and Axmacher, 2011). While most studies analyzed oscillatory activity during single-list learning (Fell et al., 2001; Summerfield and Mangels, 2005), the current experiment went one step further, investigating the impact of prefrontal control regions on brain synchrony during goaldirected forgetting. Using simultaneous EEG-fMRI in the first experiment, and rTMS-EEG in the second experiment, we provide evidence that the prefrontal cortex can drive a widespread reduction in neural synchrony when previously encoded information becomes obsolete. These results suggest that prefrontally mediated regulation of long-range synchrony might be a general mechanism underlying memory control.

\section{References}

Anderson MC (2005) The role of inhibitory control in forgetting unwanted memories: a consideration of three methods. In: Dynamic cognitive processes (MacLeod CM, Uttl B, eds), pp 159-190. Tokyo: Springer.

Anderson MC, Green C (2001) Suppressing unwanted memories by executive control. Nature 410:366-369.

Anderson MC, Ochsner KN, Kuhl B, Cooper J, Robertson E, Gabrieli SW, Glover GH, Gabrieli JD (2004) Neural systems underlying the suppression of unwanted memories. Science 303:232-235.

Bäuml KH, Samenieh A (2010) The two faces of memory retrieval. Psychol Sci 21:793-795.

Bäuml KH, Samenieh A (2012) Influences of part-list cuing on different forms of episodic forgetting. J Exp Psychol Learn Mem Cogn 38:366-375.

Bäuml KH, Hanslmayr S, Pastötter B, Klimesch W (2008) Oscillatory correlates of intentional updating in episodic memory. Neuroimage 41:596604.

Bäuml KH, Pastötter B, Hanslmayr S (2010) Binding and inhibition in episodic memory-cognitive, emotional, and neural processes. Neurosci Biobehav Rev 34:1047-1054.

Bjork EL, Bjork RA (1996) Continuing influences of to-be-forgotten information. Conscious Cogn 5:176-196.

Bjork RA (1970) Positive forgetting: the noninterference of items intentionally forgotten. J Verb Learn Behav 9:255-268.

Bjork RA (1989) Retrieval inhibition as an adaptive mechanism in human memory. In: Varieties of memory and consciousness: essays in honor of Endel Tulving (Roediger HL, ed), pp 309-330. Hillsdale, NJ: Erlbaum.

Blair RC, Karniski W (1993) An alternative method for significance testing of waveform difference potentials. Psychophysiology 30:518-524.

Buzsáki G (2006) Rhythms of the brain, Ed 1. New York: Oxford UP.

Buzsáki G (2010) Neural syntax: cell assemblies, synapsembles, and readers. Neuron 68:362-385.

Chen R, Classen J, Gerloff C, Celnik P, Wassermann EM, Hallett M, Cohen LG (1997) Depression of motor cortex excitability by low-frequency transcranial magnetic stimulation. Neurology 48:1398-1403. 
Coltheart M (1981) The MRC psycholinguistic database. Q J Exp Psychol 33A:497-505.

Conway MA, Fthenaki A (2003) Disruption of inhibitory control of memory following lesions to the frontal and temporal lobes. Cortex 39:667-686.

Debener S, Mullinger KJ, Niazy RK, Bowtell RW (2008) Properties of the ballistocardiogram artefact as revealed by EEG recordings at 1.5, 3 and $7 \mathrm{~T}$ static magnetic field strength. Int J Psychophysiol 67:189-199.

Delorme A, Makeig S (2004) EEGLAB: an open source toolbox for analysis of single-trial EEG dynamics including independent component analysis. J Neurosci Methods 134:9-21.

Depue BE (2012) A neuroanatomical model of prefrontal inhibitory modulation of memory retrieval. Neurosci Biobehav Rev 36:1382-1399.

Depue BE, Curran T, Banich MT (2007) Prefrontal regions orchestrate suppression of emotional memories via a two-phase process. Science 317:215-219.

Fell J, Axmacher N (2011) The role of phase synchronization in memory processes. Nat Rev Neurosci 12:105-118.

Fell J, Klaver P, Lehnertz K, Grunwald T, Schaller C, Elger CE, Fernández G (2001) Human memory formation is accompanied by rhinalhippocampal coupling and decoupling. Nat Neurosci 4:1259-1264.

Field DJ, Hayes A, Hess RF (1993) Contour integration by the human visual system: evidence for a local "association field." Vision Res 33:173-193.

Fisher NI (1993) Statistical analysis of circular data. Cambridge, UK: Cambridge UP.

Fries P (2005) A mechanism for cognitive dynamics: neuronal communication through neuronal coherence. Trends Cogn Sci 9:474-480.

Friston KJ, Holmes AP, Worsley KJ, Poline JB, Frith C, Frackowiak RSJ (1995) Statistical parametric maps in functional imaging: a general linear approach. Hum Brain Mapp 2:189-210.

Friston KJ, Buechel C, Fink GR, Morris J, Rolls E, Dolan RJ (1997) Psychophysiological and modulatory interactions in neuroimaging. Neuroimage 6:218-229.

Fuster JM (1997) Network memory. Trends Neurosci 20:451-459.

Geiselman RE, Bjork RA, Fishman DL (1983) Disrupted retrieval in directed forgetting: a link with posthypnotic amnesia. J Exp Psychol Gen 112:58-72.

Hanslmayr S, Aslan A, Staudigl T, Klimesch W, Herrmann CS, Bäuml KH (2007) Prestimulus oscillations predict visual perception performance between and within subjects. Neuroimage 37:1465-1473.

Hanslmayr S, Leipold P, Pastötter B, Bäuml KH (2009a) Anticipatory signatures of voluntary memory suppression. J Neurosci 29:2742-2747.

Hanslmayr S, Spitzer B, Bäuml KH (2009b) Brain oscillations dissociate between semantic and nonsemantic encoding of episodic memories. Cereb Cortex 19:1631-1640.

Hanslmayr S, Staudigl T, Fellner MC (2012) Oscillatory power decreases and long-term memory: the information via desynchronization hypothesis. Front Hum Neurosci 6:74.

Knoch D, Treyer V, Regard M, Müri RM, Buck A, Weber B (2006) Lateralized and frequency-dependent effects of prefrontal rTMS on regional cerebral blood flow. Neuroimage 31:641-648.

Lachaux JP, Rodriguez E, Martinerie J, Varela FJ (1999) Measuring phase synchrony in brain signals. Hum Brain Mapp 8:194-208.

Levy BJ, Anderson MC (2002) Inhibitory processes and the control of memory retrieval. Trends Cogn Sci 6:299-305.

Li X, Nahas Z, Kozel FA, Anderson B, Bohning DE, George MS (2004) Acute left prefrontal transcranial magnetic stimulation in depressed patients is associated with immediately increased activity in prefrontal cortical as well as subcortical regions. Biol Psychiatry 55:882-890.

Mantini D, Perrucci MG, Del Gratta C, Romani GL, Corbetta M (2007) Electrophysiological signatures of resting state networks in the human brain. Proc Natl Acad Sci U S A 104:13170-13175.

Markram H, Lübke J, Frotscher M, Sakmann B (1997) Regulation of synaptic efficacy by coincidence of postsynaptic APs and EPSPs. Science 275:213-215.

Miranda PC, Lomarev M, Hallett M (2006) Modeling the current distribution during transcranial direct current stimulation. Clin Neurophysiol 117:1623-1629.

Niazy RK, Beckmann CF, Iannetti GD, Brady JM, Smith SM (2005) Removal of FMRI environment artifacts from EEG data using optimal basis sets. Neuroimage 28:720-737.

Nyberg L, Andersson M, Forsgren L, Jakobsson-Mo S, Larsson A, Marklund P, Nilsson LG, Riklund K, Bäckman L (2009) Striatal dopamine D2 binding is related to frontal BOLD response during updating of long-term memory representations. Neuroimage 46:1194-1199.

Oostenveld R, Fries P, Maris E, Schoffelen JM (2011) FieldTrip: open source software for advanced analysis of MEG, EEG, and invasive electrophysiological data. Comput Intell Neurosci 2011:156869.

Pastötter B, Bäuml KH (2007) The crucial role of postcue encoding in directed forgetting and context-dependent forgetting. J Exp Psychol Learn Mem Cogn 33:977-982.

Pastötter B, Bäuml KH (2010) Amount of postcue encoding predicts amount of directed forgetting. J Exp Psychol Learn Mem Cogn 36:54-65.

Pastötter B, Bäuml KH, Hanslmayr S (2008) Oscillatory brain activity before and after an internal context change-evidence for a reset of encoding processes. Neuroimage 43:173-181.

Pastötter B, Schicker S, Niedernhuber J, Bäuml KH (2011) Retrieval during learning facilitates subsequent memory encoding. J Exp Psychol Learn Mem Cogn 37:287-297.

Rosanova M, Casali A, Bellina V, Resta F, Mariotti M, Massimini M (2009) Natural frequencies of human corticothalamic circuits. J Neurosci 29:7679-7685.

Sahakyan L, Delaney PF (2003) Can encoding differences explain the benefits of directed forgetting in the list method paradigm? J Mem Lang 48:195-206

Sahakyan L, Kelley CM (2002) A contextual change account of the directed forgetting effect. J Exp Psychol Learn Mem Cogn 28:1064-1072.

Sederberg PB, Gauthier LV, Terushkin V, Miller JF, Barnathan JA, Kahana MJ (2006) Oscillatory correlates of the primacy effect in episodic memory. Neuroimage 32:1422-1431.

Silvanto J, Muggleton N, Walsh V (2008) State-dependency in brain stimulation studies of perception and cognition. Trends Cogn Sci 12:447-454.

Summerfield C, Mangels JA (2005) Coherent theta-band EEG activity predicts item-context binding during encoding. Neuroimage 24:692-703.

Thut G, Pascual-Leone A (2010) A review of combined TMS-EEG studies to characterize lasting effects of repetitive TMS and assess their usefulness in cognitive and clinical neuroscience. Brain Topogr 22:219-232.

Underwood BJ (1978) Recognition memory as a function of length of study list. Bull Psychon Soc 12:89-91.

Varela F, Lachaux JP, Rodriguez E, Martinerie J (2001) The brainweb: phase synchronization and large-scale integration. Nat Rev Neurosci 2:229-239.

Ward NS, Bestmann S, Hartwigsen G, Weiss MM, Christensen LO, Frackowiak RS, Rothwell JC, Siebner HR (2010) Low-frequency transcranial magnetic stimulation over left dorsal premotor cortex improves the dynamic control of visuospatially cued actions. J Neurosci 30:9216-9223. 